\title{
ARTICLE \\ Sex and strain differences in dynamic and static properties of the mesolimbic dopamine system
}

\author{
Maria Teresa Rivera-Garcia ${ }^{1}$, Aqilah M. McCane $\mathbb{D}^{1}$, Tara G. Chowdhury ${ }^{1,2}$, Kathryn G. Wallin-Miller ${ }^{1}$ and Bita Moghaddam $^{1}$
}

\begin{abstract}
Sex is a biological variable that contributes to the incidence, clinical course, and treatment outcome of brain disorders. Chief among these are disorders associated with the dopamine system. These include Parkinson's disease, ADHD, schizophrenia, and mood disorders, which show stark differences in prevalence and outcome between men and women. In order to reveal the influence of biological sex as a risk factor in these disorders, there is a critical need to collect fundamental information about basic properties of the dopamine system in males and females. In Long Evans rats, we measured dynamic and static properties related to the mesolimbic dopamine system. Static measures included assessing ventral tegmental area (VTA) dopamine cell number and volume and expression of tyrosine hydroxylase and dopamine transporter. Dynamic measures in behaving animals included assessing (1) VTA neuronal encoding during learning of a cue-action-reward instrumental task and (2) dopamine release in the nucleus accumbens in response to electrical stimulation of the VTA, vesicular depletion of dopamine, and amphetamine. We found little or no sex difference in these measures, suggesting sexual congruency in fundamental static and dynamic properties of dopamine neurons. Thus, dopamine related sex-differences are likely mediated by secondary mechanisms that flexibly influence the function of the dopamine cells and circuits. Finally, we noted that most behavioral sex differences had been reported in Sprague-Dawley rats and repeated some of the above measures in that strain. We found some sex differences in those animals highlighting the importance of considering strain differences in experimental design and result interpretation.
\end{abstract}

Neuropsychopharmacology (2020) 45:2079-2086; https://doi.org/10.1038/s41386-020-0765-1

\section{INTRODUCTION}

Dopamine neurotransmission is fundamental to motivation, movement, and cognition [1, 2] and may be involved in several neuropsychiatric disorders including attention deficit hyperactivity disorder (ADHD), Parkinson's disease, major depression, schizophrenia, and substance use disorder [3]. Although these disorders are characterized by significant sex differences $[4,5]$, our current knowledge of the dopamine system is based primarily on data from male animal models. Existing literature incorporating females has often emphasized the role of estrous cycle and sex hormones [6-8]. However, many dopamine-related disorders, such as ADHD or Parkinson's disease, occur long before sexual maturation or after menopause. Thus, uncovering the fundamental properties of dopamine neurons in male and female subjects is critical to our understanding and treatment of psychiatric disease.

Recent studies have reported a lack of sexual dimorphism in the input and output relationships of dopamine neurons in the ventral tegmental area (VTA), as well as in their intrinsic electrophysiological properties [9]. Sex differences, however, may exist in the functional dynamics of this system. In particular, sex differences in motivated behaviors and in the response to psychostimulant drugs suggest that dopamine function differs in males and females [10-12].

Here, we characterized fundamental static and dynamic measurements of VTA dopamine neurons in male and female Long Evans (LE) rats. Static measures included dopamine cell number and volume, tyrosine hydroxylase (TH) expression in the VTA, and dopamine transporter (DAT) density in the target region of the nucleus accumbens (NAc). Dynamic measures included VTA dopamine neuron activity during instrumental conditioning, measurement of NAc terminal dopamine release, and behavioral activation in response to: (1) electrical stimulation of VTA neurons, (2) pharmacological inhibition of vesicular monoamine transporter (VMAT2), and (3) systemic exposure to amphetamine. Our results show subtle or no sex differences in these measures in LE rats suggesting sexual congruency in fundamental static and dynamic properties of dopamine neurons. Given that most previous behavioral sex differences have been reported in SpragueDawley (SD) rats $[7,13-16]$, we repeated some of the above measures in SD rats.

\section{METHODS}

No data was collected during the COVID19 pandemic.

Animals

Adult male and female LE and SD rats were bred in-house or purchased from Charles River Laboratories. Rats were pair-housed until surgery under humidity and temperature-controlled conditions using a 12-h reverse light/dark cycle with lights off at 9:00 am. All experimental procedures were performed during the dark phase, in accordance with the National Institute of Health's Guide

\footnotetext{
${ }^{1}$ Department Behavioral Neuroscience, Oregon Health and Science University, Portland, OR, USA

Correspondence: Maria Teresa Rivera-Garcia (riveragm@ohsu.edu)

${ }^{2}$ Present address: Department of Psychiatry, Oregon Health and Science University, Portland, OR, USA
}

Received: 5 March 2020 Revised: 4 July 2020 Accepted: 8 July 2020

Published online: 14 July 2020 
to the Care and Use of Laboratory Animals and were approved by the Oregon Health and Science University Institutional Animal Care and Use Committee.

\section{Drugs}

Reserpine $(5 \mathrm{mg} / \mathrm{kg})$ and amphetamine $(2 \mathrm{mg} / \mathrm{kg})$ were purchased from Sigma-Aldrich Inc. (St. Louis, MO), dissolved in $0.9 \%$ sterile saline and injected intraperitoneally (i.p.; $1 \mathrm{ml} / \mathrm{kg}$ ).

Immunohistochemistry and light microscopy

LE and SD (4-5 males and females per strain) naive rats were perfused according to Gage and colleagues [17]. Brains were frozen and cut at $40-\mu \mathrm{m}$ thickness using a cryostat (CM1950; Leica). Primary antibodies targeting DAT (1:100, Bioss bs-1714R) and TH (1:1000; Abcam ab76442) were used as suggested by manufacturers. Alexa Fluor-conjugated secondary antibodies (1:500; Abcam ab150081 or ab150176) were used to visualize the protein of interest with fluorescent staining. A Zeiss Axiovert 200 microscope (Zeiss) with an Axiocam camera and Apotome II instrument with grid-based optical sectioning was used to image midbrain TH-labeled cells (red channel) in the VTA or DAT staining (green channel) in the NAc. Each image was acquired with the $\times 20$ objective and the Zen 2 software (Zeiss) generated a Z-stack scan series, consisting of $251-\mu \mathrm{m}$ scans, which provided a total volume of $120 \times 120 \times 25 \mu^{3}$ per image stack. For fluorescence quantification, three alternating sections were taken per animal and both hemispheres were imaged but only one hemisphere was analyzed. In all cases, values from those three images were averaged and we obtained a single value per animal for each parameter. In order to collect the same sections, we considered a range of $4.9-5.5 \mathrm{~mm}$ for VTA and $0.9-1.40 \mathrm{~mm}$ for NAc in the AP axis with respect to Bregma. Imaris software (v.9.2.0; Bitplane) was used for the image processing and quantification of the parameters of interest.

\section{Surgery}

All surgeries were performed under isoflurane anesthesia between postnatal days 65-70. For microdialysis experiments, animals were implanted with guide cannulas in the NAC $(A P=+1.2 \mathrm{~mm} \mathrm{ML}=$ $1.1 \mathrm{~mm}$ from Bregma and $\mathrm{DV}=-6.0 \mathrm{~mm}$ from skull for males and $\mathrm{AP}=+1.2 \mathrm{~mm} \mathrm{ML}=1.1 \mathrm{~mm}$ from Bregma and $\mathrm{DV}=-6.0 \mathrm{~mm}$ from skull for females) and a bipolar stimulating electrode in the VTA (AP $=-5.3 \mathrm{~mm} \mathrm{ML}=0.9 \mathrm{~mm}$ from Bregma and DV $=-8.3$ $\mathrm{mm}$ from skull for males and $\mathrm{AP}=-5.3 \mathrm{ML}=0.7$ from Bregma and $D V=-8.3$ from skull for females). For electrophysiology experiments, home-built 50- $\mu \mathrm{m}$ Teflon-coated 8-wire microelectrode arrays were implanted in the VTA $(A P=-5.3 \mathrm{~mm} \mathrm{ML}=0.7$ $\mathrm{mm}$ from Bregma and DV $=-7.9 \mathrm{~mm}$ from skull for males and females).

\section{Electrophysiology and behavior}

These experiments were conducted in LE rats $(6$ male and 7 female) over 7 days.

Electrophysiological recordings of single-unit spiking activity were conducted during conditioning (described below) as reported previously [18]. Spikes were amplified at 1000x gain, digitized at $40 \mathrm{kHz}$, and single-unit data was band-pass filtered at $300 \mathrm{~Hz}$ by the recording software (Plexon, Dallas, TX). Single units were isolated in Kilosort as described previously [19].

After surgical recovery, rats were mildly food restricted. Behavioral training began with food-magazine training, during which sucrose pellets (fortified dextrose, $45 \mathrm{mg}$; Bio-Serv) were delivered at varying intervals (pseudorandom centered around 75 s) and continued until the animal consumed all of the pellets delivered. Next, rats learned to poke for an individual pellet. Each trial started with the onset of a nose-poke cue light. If the rat performed a nose-poke, the cue light would turn off immediately and a pellet would be delivered to the now-illuminated food trough. Once the animal retrieved the pellet, the food trough light turned off and the inter-trial interval (ITI) began (pseudorandom length of ITI centered around $10 \mathrm{~s}$ ). Each session lasted $30 \mathrm{~min}$ or until the rat completed 100 trials.

\section{Microdialysis and electrical stimulation}

One week after surgery, microdialysis experiments in LE (5 males and 6 females) or SD (8 males and 8 females) freely moving rats were conducted as described previously [20]. Dialysis probes (CMA Microdialysis) with an active membrane length of $2 \mathrm{~mm}$ were inserted into the guide cannula, Ringer's solution (in $\mathrm{mM}: 37.0$ $\mathrm{NaCl}, 0.7 \mathrm{KCl}, 0.25 \mathrm{MgCl}_{2}$, and $3.0 \mathrm{CaCl}_{2}$ ) was perfused at a flow rate of $2.0 \mu \mathrm{L} / \mathrm{min}$. After $60 \mathrm{~min}$ of stabilization, dialysis samples (20 min each) were collected and immediately injected into a high performance liquid chromatography system with electrochemical detection of dopamine, as described before [21]. After three consecutive stable baseline samples the treatment (electrical stimulation or drug administration) was delivered.

Locomotion was simultaneously measured using a home cage activity detection system (Hamilton-Kinder, LLC, Poway, CA). Beam-breaks activity were tracked using the Kinder Scientific MotorMonitor program.

For VTA cells stimulation, the electrode was connected to a stimulator (Grass Technologies) and a phasic burst stimulation protocol was applied (1-ms pulses at $100 \mathrm{~Hz}$ for $200 \mathrm{~ms}$, inter-burst interval: $500 \mathrm{~ms}$ for $20 \mathrm{~min}$ at $6 \mu \mathrm{A}$ ) [20].

For reserpine and amphetamine experiments, independent cohorts of LE rats (5 males and 5 females) were used, following same procedures for surgery, microdialysis and histology as described for other cohorts.

\section{Histology}

At the end of behavioral experiments, all rats were perfused, and brains were cryoprotected and sectioned as described above. Probe and electrodes placement was determined during sectioning of the brain tissue and data from animals with incorrect placement were not included in the final analysis (Fig. S1).

\section{Data analysis}

Microdialysis data were expressed as percent of baseline value, defined as the mean of three consecutive samples obtained before any electrical or pharmacological manipulation. Locomotor activity data were expressed as the number of infrared beam breaks within a 20-min bin and expressed as basic movements (total $X / Y$ breaks). Statistical analyses of these dependent measures was conducted using two-factor repeated-measures ANOVA with sex as the between-subjects factor and time as the within-subjects factor.

The immunohistochemistry data were expressed as standardized fluorescence from tissue surfaces. For immunohistochemistry analysis, independent $t$-tests were used to compare values between male and female groups.

For electrophysiology data, putative dopamine units were classified based on waveform duration $(>1.2 \mathrm{~ms})$ and firing rate $(<12$ spikes/s) [18]. Recordings with no putative dopamine cells were excluded from analyses, yielding a total of 82 data sets $(N=$ $44 /$ male, $N=38 /$ female). Baseline firing rate was taken as an average firing rate during the baseline window $(2 \mathrm{~s}$, beginning $2.5 \mathrm{~s}$ before the onset of the cue). Firing rates during instrumental performance were analyzed collapsed across sessions 2-8, after behavior was learned. Data were divided into three 500 millisecond (ms) epochs, before, during and after each event (cue, action, and reward delivery) and changes in firing rate were assessed via mixed-design ANOVA (between subjects factor: sex; within subjects factor: epoch). Significant main effects of epoch were followed up with Dunnett's post hoc comparing firing rate during the three epochs surrounding each event (before, during, and after the event of interest). Bonferroni corrected $t$-tests were 
A

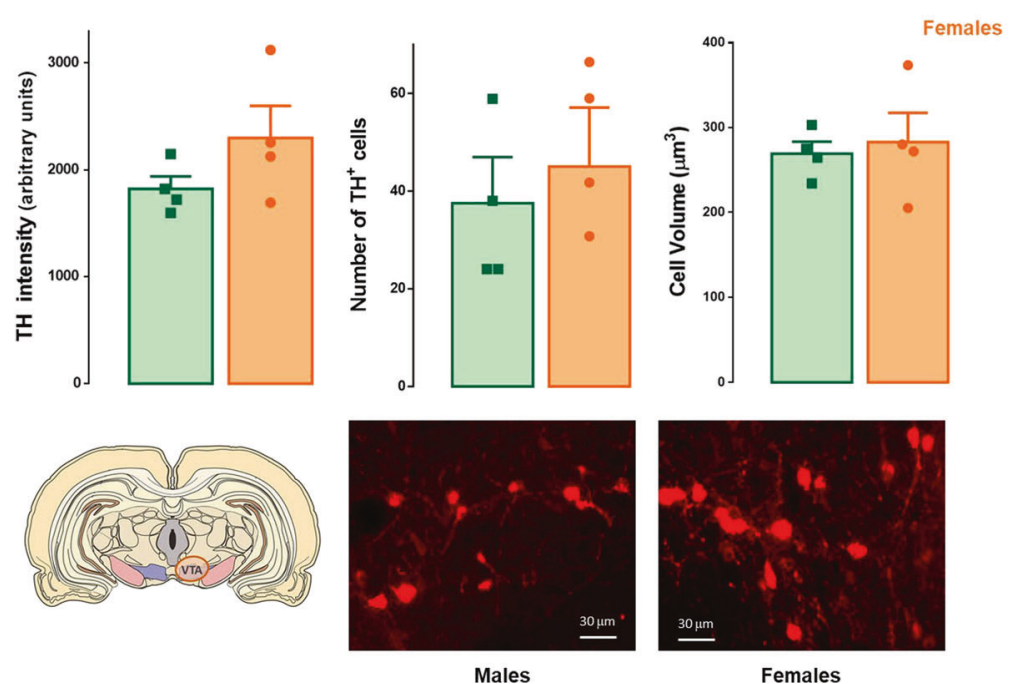

B
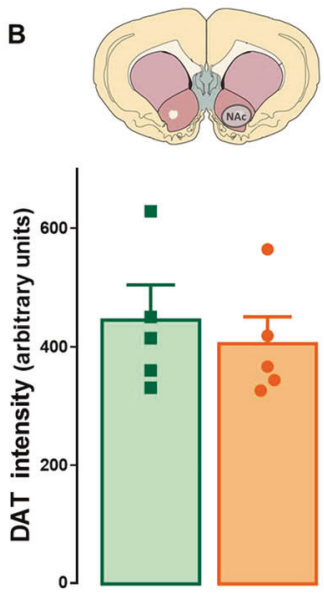

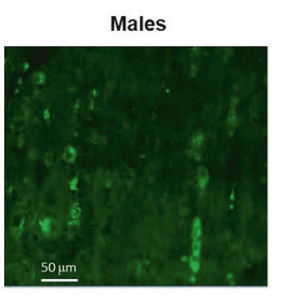

Females

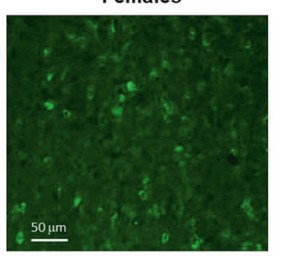

Fig. 1 Analysis of sex differences in static properties of the dopamine system in LE rats. a there is no difference between male (green bars) and female (orange bars) rats in TH expression $\left(t_{6}=1.55, p=0.19\right)$, number $\left(t_{6}=0.49, p=0.64\right)$ or size $\left(t_{6}=0.36, p=0.73\right)$ of TH+ cells on the VTA ( $n=4$ per group). Representative images of TH staining of the VTA in male and female rats. b No significant sex difference in DAT expression in the NAc $\left(t_{8}=0.53, p=0.61 ; n=5\right.$ per group). Data are expressed as mean \pm SEM.

performed to determine if sex influenced changes in firing rates during events. In all cases, data are expressed as mean \pm SEM, values of $p \leq 0.05$ were considered statistically significant, and values of $0.05<p<0.1$ were considered trending.

\section{RESULTS}

Dopamine system characterization: cell number and size, $\mathrm{TH}$ and DAT expression

We found no difference between sexes in TH expression, number, or size of dopamine cells in male and female LE rats $\left(t_{6}=1.55, p=\right.$ $0.19 ; t_{6}=0.49, p=0.64 ; t_{6}=0.36, p=0.73$, respectively; Fig. 1 ). Because DAT regulates dopamine availability downstream of the VTA, we also analyzed the expression of this protein in the NAc. As depicted in Fig. 1b, our analysis revealed similar DAT expression in the NAC of male and female rats $\left(t_{8}=0.53, p=0.61\right)$. In an independent cohort of older LE rats that had been tested extensively for operant behaviors, we found sex differences with females having a higher number of TH + cells $\left(t_{11}=5.93, p<0.05\right)$ and greater intensity of TH expression $\left(t_{11}=3.93, p<0.05\right)$ in VTA (Fig. S2).

Behavior and VTA neuron activity during instrumental learning Unit activity was recorded from the VTA of male $(n=6)$ and female $(n=7)$ rats while they learned an instrumental behavior (Fig. 2). Both sexes equally learned the task as evident by a decrease in latency to poke (main effect of session: $F(7,54)=3.86$, $p=0.002)$. Latency to retrieve a reward did not significantly differ by session (main effect of session: $F(7,54)=1.84, p=0.09$ ). Although females completed more trials than males (main effect of sex: $F(1,2)=26.32, p=0.04$; Fig. $2 b$ ), sex did not influence learning (latency to poke, sex $\times$ session: $\mathrm{F}(7,54)=0.35, p=0.93$; latency to retrieve reward, $\operatorname{sex} \times$ session: $\mathrm{F}(7,54)=0.88, p=0.53$ ). In addition, there was no significant sex-related difference across training in the baseline firing rate of putative dopamine cells $\left(t_{507}=-1.21, p=0.22\right.$; Fig. $\left.2 \mathrm{~d}\right)$. We next asked whether firing rate changed during the event (epoch) of interest and whether this change was influenced by sex. The collapsed data from sessions 2-8 revealed a phasic response to cue presentation in both sexes (main effect of epoch: $F(2,276)=30.33, p<0.001$ ), where the neurons from both groups became activated in a similar magnitude (Bonferroni post hoc, $p=0.09$; Fig. 2e). There was also an increase in neuronal activity within the $0.8 \mathrm{~s}$ after the action (main effect of epoch: $\mathrm{F}(2,276)=10.36, p<0.001)$ with no difference between groups (Bonferroni post hoc, $p=3.09$; Fig. 3e). Finally, firing rate increased in response to reward delivery (main effect of epoch: $F(2,220)=3.56, p=0.03$ ) in both groups (Bonferroni post hoc, $p=0.26$; Fig. 2e), suggesting that dopamine neuron phasic activity during learning and performance of an instrumental task is similar in males and females.

Extracellular dopamine dynamics in behaving animals

We next investigated sex differences in neurochemical dynamics of the mesolimbic circuit. In freely moving animals, we used electrical stimulation to drive VTA cells and measured dopamine release in the NAc. There were no sex differences in mean baseline dopamine levels (males: $1.50 \pm 0.50$, females: $0.79 \pm 0.25 \mathrm{fmol} / \mu \mathrm{L}$; $t_{12}=-1.46 ; \quad p=0.17$ ). As expected, phasic burst electrical stimulation increased dopamine levels relative to baseline in both groups (time: $F(9,81)=16.16, p<0.0001$ ), with higher levels in males than females (sex: $F(1,9)=5.34, p=0.05$; time $\times$ sex interaction: $F(9,81)=2.69, p=0.01$; Fig. 3b). VTA electrical stimulation induced a transient increase in locomotor activity ( $F$ $(9,81)=4.73, p<0.0001)$ with no significant difference between groups (sex: $F(1,9)=1.80, p=0.21$; time $\times$ sex interaction: $F(9,81$ ) $=0.28, p=0.98$; Fig. 3b).

Pharmacological tools were used next to probe potential differences in the release and storage dynamics of the dopamine system. Reserpine (5 mg/kg), which blocks VMAT [22], produced similar results in both sexes (sex: $\mathrm{F}(1,8)=0.52, p=0.49$; time $\times$ sex interaction: $F(14,112)=0.40, p=0.97$; Fig. $4 d)$. An increase in dopamine levels in the NAC (main effect of time: $F(14,112)=15.94$, $p<0.0001$ ) was followed by a decrease at sample 7. At the behavioral level, there was an initial increase followed by a decrease in locomotor activity (time: $\mathrm{F}(10,80)=5.35, p<0.0001$ ) with no difference between groups (sex: $\mathrm{F}(1,8)=0.10, p=0.35$; time $\times$ sex interaction: $F(10,80)=0.28, p=0.98$; Fig. 3d). Baseline dopamine levels in this cohort were similar in both sexes (in fmol/ $\mu \mathrm{L}, 0.72 \pm 0.17$ for males and $0.67 \pm 0.30$ for females, $t_{8}=-0.14$; $p=0.28)$.

In reserpine-treated animals, once dopamine levels stabilized, we used amphetamine ( $2 \mathrm{mg} / \mathrm{kg}$ i.p.) to probe differences in 


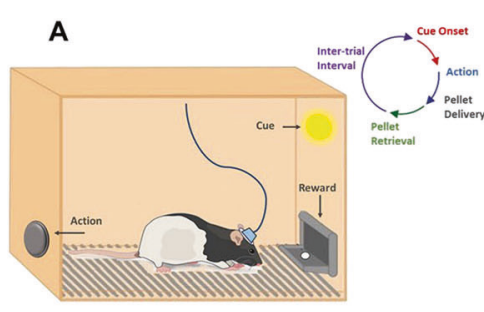

C
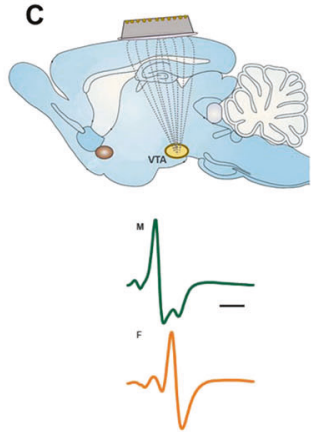

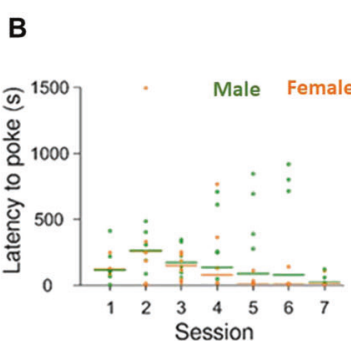

D

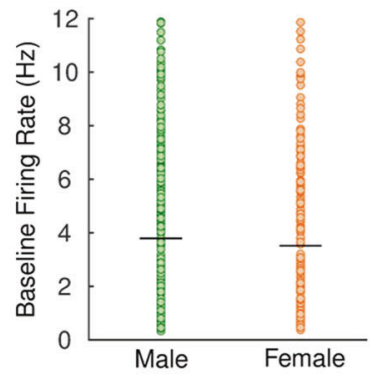

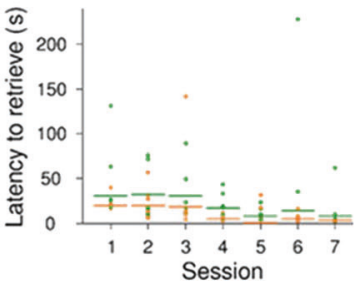

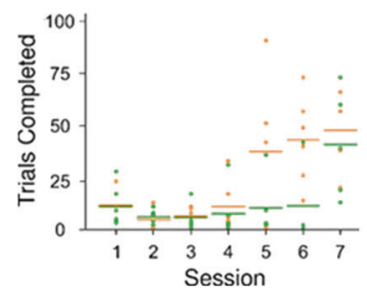

E

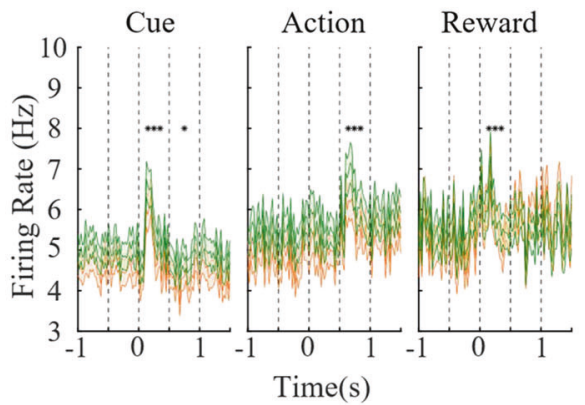

Fig. 2 Analysis of sex differences in the dynamics of dopamine system. a Schematic representation of VTA recording neuron activity during an instrumental task. The task was performed in an operant box with one nose-poke hole on one wall and a food trough on the opposite wall. Trials began when a light turned on in the nose-poke hole (cue), if the rat nose poked into the hole (action), the light turned off and a sugar pellet was immediately delivered to the now illuminated food trough. When the rat retrieved the sugar pellet, the food trough light turned off and a 10-s inter-trial interval (ITI) was triggered, followed immediately by the next trial. b As expected, across sessions there was a decrease in latency to poke and retrieve without significant differences between groups (latency to poke, sex $\times \operatorname{session:~} F(7,54)=0.35, p=0.93$; latency to retrieve reward, sex $\times$ session: $F(7,54)=0.88, p=0.53$ ), and a significant increase in completed trials favoring females (main effect of sex: $F(1,2)=26.32, p=0.04)$. c Schematic representation of wire array connectors implanted in the VTA and representative waveforms of putative dopaminergic neurons of male and females. $\mathbf{d}$ There was no significant sex difference across behavioral training in base line firing rate of putative dopaminergic cells $\left(t_{507}=-1.21, p=0.22\right)$. e Collapsed data showed an increase in firing rate of dopaminergic neurons after cue $(\mathrm{F}(2,276)=30.33, p<0.001$; Bonferroni post hoc, $p=0.09)$, action $(\mathrm{F}(2,276)=10.36, p<0.001$; Bonferroni post hoc, $p=3.09)$ and reward delivery $(\mathrm{F}(2,220)=3.56, p=0.03$; Bonferroni post hoc, $p=0.26)$ without differences between groups. ${ }^{*} p<0.05,{ }^{* * *} p<0.001$, Dunnett's post hoc.

vesicular and cytosolic dopamine pools [22]. As illustrated in Fig. 3d, amphetamine significantly increased dopamine levels (main effect of time: $F(9,72)=8.90, p<0.0001$ ) without differences between groups (sex: $\mathrm{F}(1,8)=0.06, p=0.81$; time $\times$ sex interaction: $F(9,72)=0.12, p=0.99)$. As expected, rats showed hyperactivity after amphetamine injection (main effect of time: $F(9,72)=7.22, p<0.0001$ ) but no sex difference (sex: $\mathrm{F}(1,8)=0.43, p=0.53$; time $\times$ sex interaction: $\mathrm{F}(9,72)=0.35, p=$ 0.95; Fig. 3d).

Strain differences

Since these results contrasted several previous reports describing sex differences in the dopamine system and dopamine-related behaviors in SD rats [13,14,23], we sought to analyze the potential influence of rat strain on some of the above measures. Specifically, we compared VTA dopamine cell parameters, DAT expression in the NAc, and stimulated-dopamine release in male and female SDs.

In SD rats we found significant sex differences in DAT expression in the NAc $\left(t_{6}=2.42 ; p=0.03 ;\right.$ Fig. $\left.4 a\right)$. There was a significant strain differences in DAT expression $(F(1,14)=128.9$; $p<0.0001$, Table S1). Although no significant sex differences in $\mathrm{TH}$ expression, cell number, and cell size $\left(t_{6}=1.006 ; p=0.35 ; t_{6}=1.8\right.$; $p=0.06 ; t_{6}=1.05 ; p=0.334$, respectively; Fig. $4 \mathrm{~b}$ ) were found, SD males showed a consistent trend toward higher values in all immunohistochemical analyses of VTA. Moreover, SD rats exhibited a significant sex difference in basal NAc dopamine levels. Mean values (in $\mathrm{fmol} / \mu \mathrm{L}$ ) were $0.77 \pm 0.17$ for males and $0.32 \pm 0.05$ for females $\left(t_{14}=2.25 ; p=0.04\right)$. There was also a sex difference in evoked-dopamine release after VTA phasic burst stimulation, since males showed a three-fold increase in dopamine level over baseline relative to females (main effect of sex: $\mathrm{F}(1,14)=11.59 ; p=0.004$; time $\times$ sex interaction: $\mathrm{F}(9,126)=6.18$, $p<0.0001$; Fig. 4d). VTA stimulation increased locomotor activity (main effect of time: $\mathrm{F}(9,126)=52.62, p<0.0001$ ) similarly in both groups (sex: $F(1,14)=0.47, p=0.50$; time $\times$ sex interaction: $\mathrm{F}(9,126)=1.02, p=0.43$; Fig. $4 \mathrm{~d})$.

While we did not find a strain difference in dopamine release, the two-way ANOVA revealed a significant strain difference in baseline dopamine contents and baseline locomotion (Table S1).

\section{DISCUSSION}

We compared several key characteristics of the dopamine system in male and female LE and SD rats. In LE rats, we found small sex differences in evoked NAc dopamine release in behaving rats and no sex differences in dopamine cell size or number, DAT or TH expression, or firing rate of dopamine neurons during instrumental conditioning. SD rats, on the other hand, exhibited significant sex differences in some of these measures. These findings have important implications for experimental design and data interpretation from animal models.

Sex differences in the dopamine system

The dopamine system has been associated with multiple brain disorders including ADHD, addiction, schizophrenia, mood disorders and Parkinson's disease. These disorders show sex differences in their age of onset, prevalence, symptomatology and response to treatment $[4,5]$. But while dopamine transmission is implicated in the pathology of these disorders, its role in sexmediated differences remains unclear. 

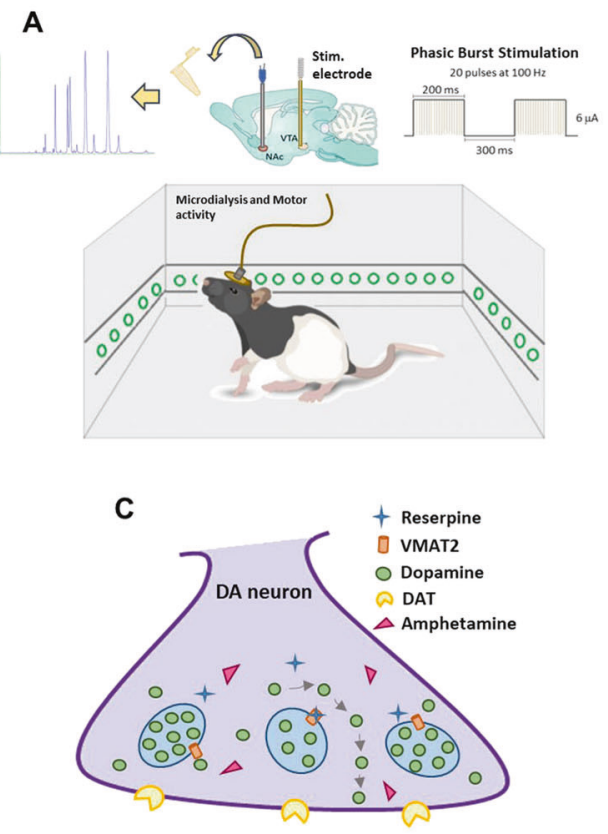
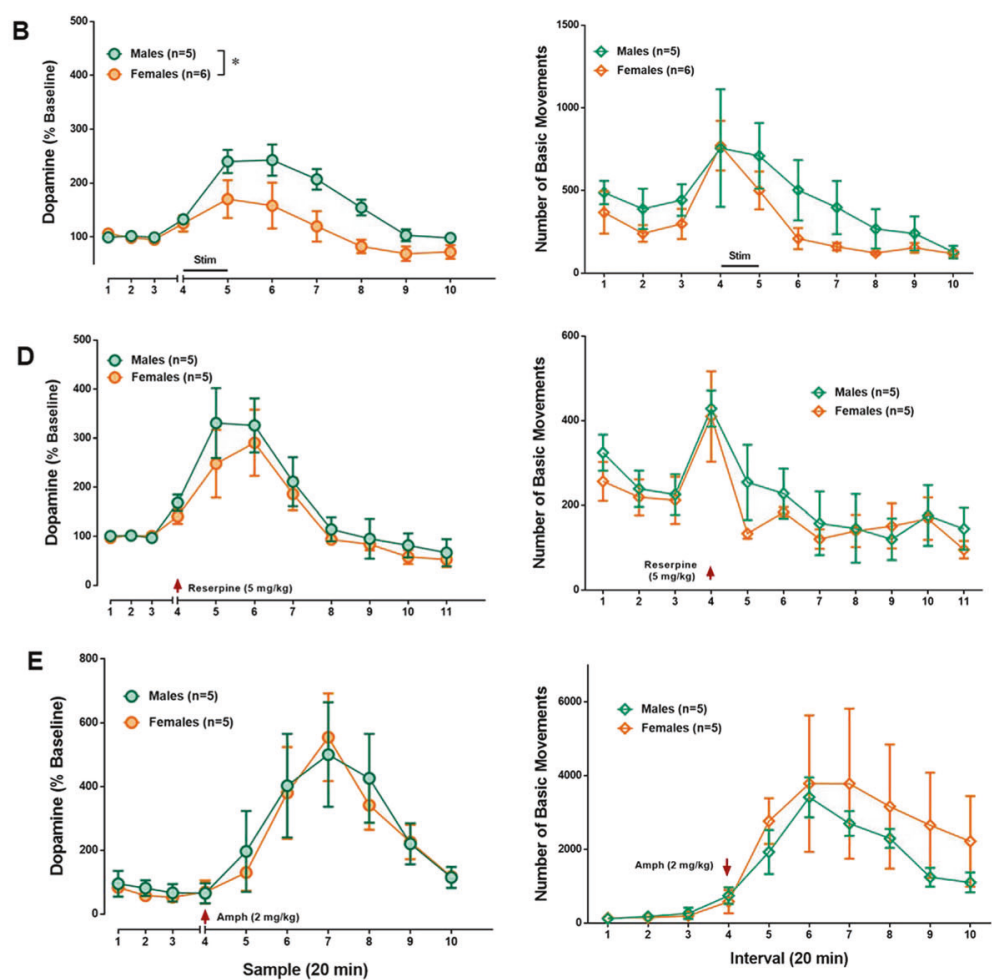

Fig. 3 Sex differences in stimulated dopamine release in the NAc. a Schematic illustrating simultaneous measure of dopamine in the NAc via microdialysis probe, VTA electrical stimulation and recording of locomotor activity on freely moving animals. The VTA was stimulated using a protocol consisting of bursts of $1 \mathrm{~ms}$ pulses delivered at $100 \mathrm{~Hz}$ for $200 \mathrm{~ms}$, with an inter-burst interval of $500 \mathrm{~ms}$ and an amplitude of $6 \mu \mathrm{A}$, for 20 min. b VTA electrical stimulation produced a significant increase in dopamine levels relative to baseline in both groups (two-way ANOVA $\mathrm{F}(9,81)=16.16, p<0.0001$ ); however, males released more dopamine compared with females (two-way ANOVA F $(1,9)=5.34, p=0.05)$. VTA stimulation induced a significant increase in locomotion which was similar between groups (two-way ANOVA F $(1,9)=1.80, p=0.21$. c Diagram of dopaminergic terminal. Reserpine blocks vesicular monoamine transporter (VMAT) and induces dopamine depletion. Amphetamine gets into the nerve terminal and releases newly synthetized dopamine. The dopamine transporter (DAT) uptakes dopamine from the extracellular space into the terminal. d Reserpine $(5 \mathrm{mg} / \mathrm{kg}$ i.p.) administration evoked a large increase in dopamine dialysates (two-way ANOVA F $(10,80)=$ $18.11, p<0.0001$ ) without difference between males and females (two-way ANOVA $\mathrm{F}(1,8)=0.55, p=0.48$ ). Reserpine injection induced a transitory increase in locomotor activity without difference between groups (two-way ANOVA $\mathrm{F}(1,8)=0.10, p=0.35$ ). e Amphetamine injection ( $2 \mathrm{mg} / \mathrm{kg}$ i.p.) produced a significant increase in dopamine release (two-way ANOVA F $(9,72)=8.90, p<0.0001)$ and locomotion (twoway ANOVA $F(9,72)=7.22, p<0.0001$ ) but no sex differences in neurochemical or behavioral amphetamine-induced effects (two-way ANOVA $\mathrm{F}(1,8)=0.06, p=0.81$ and $\mathrm{F}(1,8)=0.43, p=0.53$. Data are expressed as mean $\pm \mathrm{SEM}$. ${ }^{*} p \leq 0.05$.

Neuroimaging studies using positron emission tomography and magnetic resonance imaging have linked sex differences in reward-related behaviors to greater activation of mesolimbic dopamine system in men than women [24, 25]. Furthermore, women are more vulnerable and responsive to the rewarding effects of psychoactive drugs than men, suggesting differences in dopaminergic activity in the NAc [10, 11, 26, 27]. These findings imply important sex differences in dopamine system function, but the methodological constraints of human studies limit the ability to manipulate the system. Of note, while several clinical studies have described sex-differences related to the dopamine system $[4,5,28,29]$, these findings have not been consistent. For example, one study has shown that women have lower striatal dopamine levels and greater dopamine turnover in the putamen relative to men [30] whereas others show greater striatal dopamine synthesis, higher concentration of the dopamine synthesis precursor L-DOPA and higher DAT density in women [31, 32].

In preclinical research, sexually dimorphic midbrain regions are reported [33], but sex differences in functional and static measures of the VTA are not well understood. Although animal models have revealed sex differences in brain function and dopamine systemrelated behaviors, the findings are not always consistent. Most of the literature has emphasized the role of gonadal hormones $[6,7,34,35]$ with majority of studies ( 90\% by our account) utilizing SD albino rats and little data available in other strains that are routinely used for circuit-oriented behavioral and electrophysiological studies.

Analysis of static measurements of dopamine system

In LE rats, our analysis of dopamine system structure revealed no significant sex differences in $\mathrm{TH}$ expression, cell number, or volume of dopamine cells in the VTA, and no sex difference in DAT expression in the NAc. Our results are consistent with a previous report describing lack of sex differences in basic organization of the dopamine system in mice [9].

In SD rats, we found significant sex differences in DAT expression in the NAc and a trend in TH expression, number, and volume of TH-positive cells. Comparison of our data with previous literature is challenging given differences in methodological approaches. For example, while some studies have described sex differences in DAT function $[15,36]$, most have focused on the pharmacological profile rather than the protein expression itself. Another example involves reports showing female SD rats had a larger number and volume of dopamine cells than males [37], while other studies have described lack of sex differences in TH cell density in the VTA of SD rats [37-39].

To date, most of the studies investigating sex differences in the dopamine system and behavior have used SD rats [7, 13-15]. This strain of rats is popular because of its docile and amenable nature. 
A

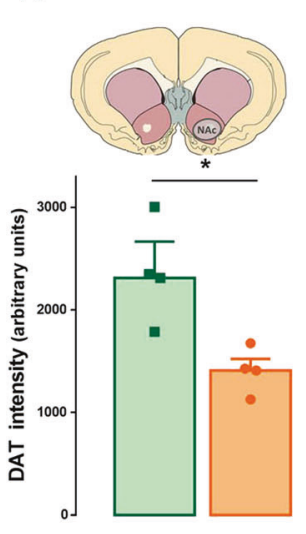

Males
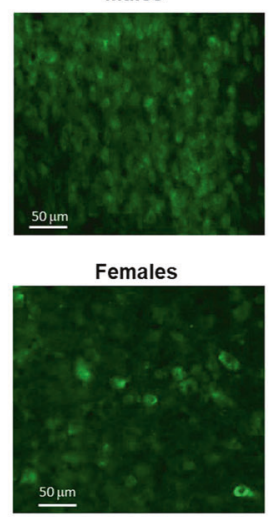

B

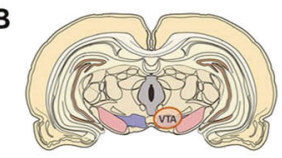

Males

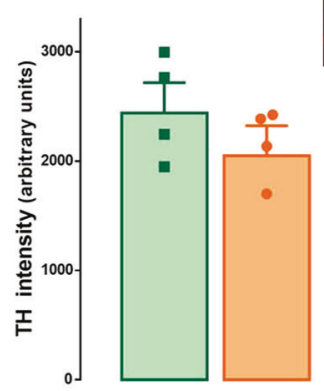

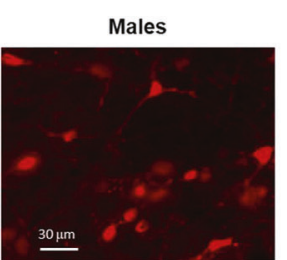

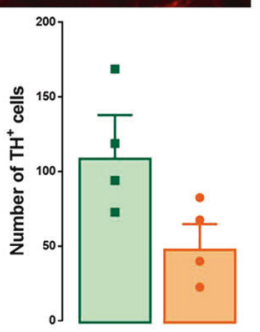

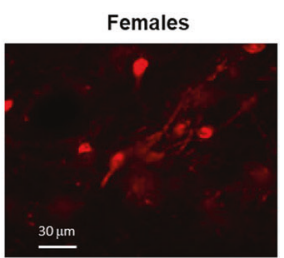

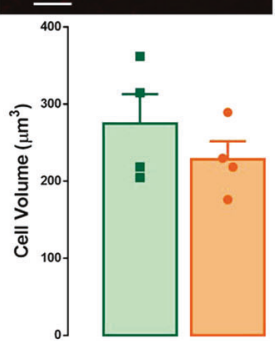

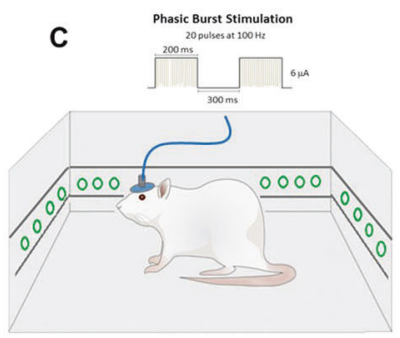

D

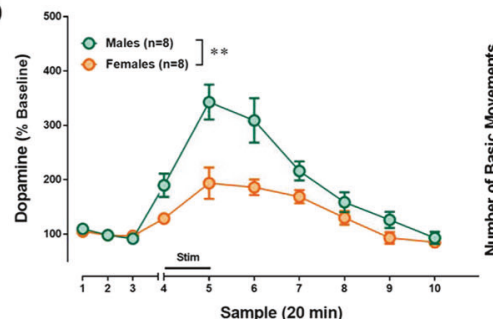

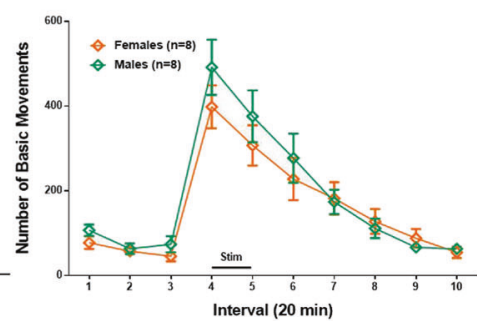

Fig. 4 Analysis of constitutive and dynamic properties of the dopamine system in SD rats. a There was a significant sex difference favoring males on DAT expression in the NAc $\left(t_{6}=2.42 ; p=0.03\right)$. b There were no sex differences in TH expression $\left(t_{6}=1.006 ; p=0.35\right)$, number $\left(t_{6}=\right.$ $1.8 ; p=0.06)$ or size $\left(t_{6}=1.05 ; p=0.334\right)$ of TH + cells in SD rats. c Schematic illustrating simultaneous VTA electrical stimulation, measurement of dopamine dialysates and locomotor activity recording of freely moving SD rats. The VTA was stimulated using a protocol consisting of bursts of $1 \mathrm{~ms}$ pulses delivered at $100 \mathrm{~Hz}$ for $200 \mathrm{~ms}$, with an inter-burst interval of $500 \mathrm{~ms}$ and an amplitude of $6 \mu \mathrm{A}$, for $20 \mathrm{~min}$. d VTA stimulation produced a higher increase in dopamine release in the NAc of males than females (two-way ANOVA F $(1,14)=11.59 ; p=0.004$ ). VTA phasic burst stimulation induced a similar increase in locomotion in both groups (two-way ANOVA F $(1,14)=0.47, p=0.50$ ). Data are expressed as mean \pm SEM. ${ }^{*} p \leq 0.05$.

Indeed, many strains have been developed specifically to model certain aspects of physiology (metabolic syndrome, tumor susceptibility) or behavioral tendencies (increased/decreased anxiety, aggression, or activity levels) [40]. While a majority of experiments investigating the dopamine system utilize SD rats, we and others prefer to use LE rats because of their superior ability to learn complex tasks [41, 42]. Overall, LE rats exhibit less anxiety, greater locomotion, and better cognitive performance than SD rats [41]. In addition, it was in LE rats that researchers developed the $\mathrm{TH}::$ Cre line, which has allowed a better understanding of dopamine system circuitry $[43,44]$. Therefore, our characterization and strain comparison of basic properties of the dopamine system is essential and powerful evidence to consider in future neurophysiological and behavioral paradigms related to dopamine function. Furthermore, given the strain differences in dopamine cell physiology, future investigations should consider strain as a variable during experimental design. Studies which seek to characterize strain differences in neural properties and function may elucidate which strains best model clinical symptomology.

Analysis of dynamic function of dopamine system In addition to the static measurements of dopamine cells, we sought to analyze sex differences in functional dynamics of the dopamine system. We compared male and female LE rats in a fixed-ratio instrumental paradigm, a basic task of associative learning, which is a fundamental aspect of more complex motivated behavior. Our data revealed similar performance between males and females during this instrumental task and no difference in learning rate. We cannot, however, discount the possibility that male and female animals used different strategies to reach the same goal.
Our results are consistent with previous studies reporting no sex differences in reward-guided associative learning [45]; however, some studies have suggested sex differences in reward-related behaviors. For example, in SD rats, females acquire drug selfadministration faster, work harder to obtain drug infusions, and take more drug during the maintenance phase under progressiveratio schedules $[10-12,26]$. However, in a different study, SD male rats acquired cocaine self-administration faster than females [34]. These different results highlight the complexity of such behavioral studies and the importance of considering strain differences. An emphasis on the direct influence of gonadal hormones rather than sex differences per se has historically been used to understand sex differences in cognition and dopamine-related behaviors $[6-8,11]$. Here, instead, we investigated sex differences in intact male and female rats for several reasons. First, recent meta-analysis studies suggest that males have the same variability compared with females in different biochemical and behavioral measures $[46,47]$. According to the authors, female subject inclusion does not require monitoring of the estrous cycle and it does not represent a source of variability in intact females' behavior, thus the most informative studies are those which explicitly compare across sex incorporating an equal number of male and female subjects $[46,47]$. Furthermore, this method more closely models the human condition, in which most males and females have intact gonads and experience natural variation in hormone levels. Importantly, our findings mainly rely on the study of sex differences under a naturalistic perspective, which is important for future analyses and interpretations of dopamine-related behaviors.

While our results suggest that sex differences in the dopamine system are more apparent in SD compared with LE rats, increasing the subject number in LE rats may potentially reveal significant 
differences. In fact, we found a statistically-significant sex difference in $\mathrm{TH}^{+}$number of cells and $\mathrm{TH}$ expression in VTA from and older and behaviorally-experienced cohort. Thus, it is possible that sex differences in VTA dopamine cell parameters are influenced not only by strain but experimental history and age.

In the cohorts used in the present study, LE rats showed little or no sex difference in dopamine efflux in response to direct VTA cell stimulation. SD rats displayed a clear sex difference in this measure. There was, however, no difference in behavioral activation in either strain suggesting that the functional consequences of dopamine-mediated activation was similar in both sexes of both strains.

We found significant strain differences in baseline dopamine contents and baseline locomotion, suggesting a hyperdopaminergic tone in LE compared with SD rats in basal conditions. This is consistent with previous reports showing that LE rats are generally more active than albino rats [41]. Furthermore, previous studies described strain differences in catecholamine contents [48] and strain differences in sensitivity to effects of non-specific dopamine agonists measured by behavioral tests [49] and autoradiography [50]. Further carefully designed studies are necessary to better describe strain differences in the dopaminergic system and its implication in sex differences.

To probe the mechanisms underlying the sex difference in dopamine release in LE rats, we challenged the system with low doses of reserpine and amphetamine. These pharmacological manipulations, albeit not perfect, served as tools to test sex differences in dopamine concentration according to the twocompartment monoamines hypothesis, which suggests morphological and functional differences in the intraneuronal distribution of monoamines [51]. We found a similar response to reserpine in both sexes.

Unrelated to sex differences, our data shows a biphasic effect of reserpine on extracellular dopamine levels. Reserpine is an old drug with a large literature demonstrating a profound reduction in vesicular dopamine stores and ultimately a reduction in impulse flow dependent dopamine release [52-54]. While our results of a delayed decrease in dopamine levels is expected, the immediate increase may appear inconsistent with older literature. It is important to underscore that the older work mostly investigated the impact of reserpine in vitro and/or in vivo after 18-36 $\mathrm{h}$ [5557] rather than short-term effects. Other in vivo reports, including work from our lab [58], that did not observe an immediate increase in extracellular levels of dopamine after reserpine used anesthetized preparations or awake animals under potentially stressful conditions such as performing experiments $24 \mathrm{~h}$ after survival surgery and chloral hydrate injection [59]. Thus, it is possible that the absence of an immediate increase in older studies is because the tonic activity of dopamine neurons and dopamine release are differently regulated in anesthetized or stressed animals $[58,60]$. We propose that the initial increase in extracellular levels of dopamine we observed was due to accumulation of intracellular dopamine by reserpine. This can result in reverse transport of dopamine by DAT to the extracellular space [61], causing a transient surge in extracellular dopamine levels.

Unlike previous work in SD rats [7, 13, 62], we found no sex differences in amphetamine-induced dopamine release or locomotor activation in LE rats. Amphetamine-induced dopamine release is attenuated by a-methyl-p-tyrosine, which blocks the activity of the rate-limiting dopamine synthesis enzyme, tyrosine hydroxylase suggesting that it is tapping into newly synthetized dopamine [53]. While amphetamine is generally assumed to release dopamine from newly synthesized extra-vesicular cytoplasmic compartments via an impulse-independent exchange diffusion mechanism [22, 63], extracellular level increases observed here may be the result of both the newly synthetized and neurotransmitter sequestered in the cytosol. Our results suggest that some or all of these stores of dopamine are similar in male and female LE rats.

\section{Conclusions}

The current study provides valuable insight into dopamine system encoding of reward-related learning and regulation of dopamine release in males and females. Our data suggest that sex differences in dopamine-related function are secondary to complex interactions in brain networks and not necessarily the result of differences in basic "hardware" of the dopamine system. Furthermore, our data highlight the importance of understanding strain differences in anatomy and physiology of the dopamine system, along with careful characterization and comparison of variables of interest that should guide our experimental design and data interpretation on sex differences in behavioral neuroscience.

\section{FUNDING AND DISCLOSURE}

This work was supported by the National Institutes of Health grant R01 grant (MH048404) to BM. The authors declare no competing interests.

\section{AUTHOR CONTRIBUTIONS}

MTRG and BM designed neurochemistry and pharmacology experiments. MTRG performed neurochemistry experiments and immunohistochemistry experiments. TGC and BM designed electrophysiology experiments. TGC and AM collected and analyzed electrophysiology data. KWM and MTRG analyzed immunohistochemistry data. MTRG and BM wrote the paper. All authors provided feedback on the manuscript.

\section{ADDITIONAL INFORMATION}

Supplementary Information accompanies this paper at (https://doi.org/10.1038/ s41386-020-0765-1)

Publisher's note Springer Nature remains neutral with regard to jurisdictional claims in published maps and institutional affiliations.

\section{REFERENCES}

1. Arias-Carrion O, Stamelou M, Murillo-Rodriguez E, Menendez-Gonzalez M, Poppel E. Dopaminergic reward system: a short integrative review. Int Arch Med. 2010;3:24.

2. Bjorklund A, Dunnett SB. Dopamine neuron systems in the brain: an update Trends Neurosci. 2007:30:194-202.

3. Klein MO, Battagello DS, Cardoso AR, Hauser DN, Bittencourt JC, Correa RG. Dopamine: functions, signaling, and association with neurological diseases. Cell Mol Neurobiol. 2019;39:31-59.

4. Bao AM, Swaab DF. Sex differences in the brain, behavior, and neuropsychiatric disorders. Neuroscientist. 2010;16:550-65.

5. McCarthy MM. Sex differences in the developing brain as a source of inherent risk. Dialogues Clin Neurosci. 2016;18:361-72.

6. Becker JB. Direct effect of 17B-estradiol on striatum: sex differences in dopamine release. Synapse. 1990;5:157-64.

7. Becker JB. Gender differences in dopaminergic function in striatum and nucleus accumbens. Pharm Biochem Behav. 1999;64:803-12.

8. Cummings JA, Jagannathan L, Jackson LR, Becker JB. Sex differences in the effects of estradiol in the nucleus accumbens and striatum on the response to cocaine: neurochemistry and behavior. Drug Alcohol Depend. 2014;135:22-8.

9. Chung AS, Miller SM, Sun Y, Xu X, Zweifel LS. Sexual congruency in the connectome and translatome of VTA dopamine neurons. Sci Rep. 2017;7:11120.

10. Becker JB, Hu M. Sex differences in drug abuse. Front Neuroendocrinol. 2008;29:36-47.

11. Carroll ME, Lynch WJ, Roth ME, Morgan AD, Cosgrove KP. Sex and estrogen influence drug abuse. Trends Pharm Sci. 2004;25:273-9.

12. Lynch WJ, Roth ME, Carroll ME. Biological basis of sex differences in drug abuse: preclinical and clinical studies. Psychopharmacology. 2002;164:121-37.

13. Milesi-Halle A, McMillan DE, Laurenzana M, A B-BK, Owens SM. Sex differences in $(+)$-amphetamine- and $(+)$-methamphetamine-induced behaviorla responses in male and female sprague-dawley rats. Pharm Biochem Behav. 2007;86:140-9. 
14. Rincon-Cortes M, Grace AA. Sex-dependent effects of stress on immobility behavior and VTA dopamine neuron activity: modulation by ketamine. Int J Neuropsychopharmacol. 2017;20:823-32.

15. Walker QD, Rooney MB, Wightman RM, Kuhn CM. Dopamine release and uptake are greater in female than male rat striatum as measured by fast cyclic voltammetry. Neuroscience. 2001;95:1061.

16. Roth ME, Cosgrove KP, Carroll ME. Sex differences in the vulnerability to drug abuse: a review of preclinical studies. Neurosci Biobehav Rev. 2004:28:533-46.

17. Gage GJ, Kipke DR, Shain W. Whole animal perfusion fixation for rodents. J Vis Exp. 2012;3564. https://doi.org/10.3791/3564.

18. Kim Y, Simon NW, Wood J, Moghaddam B. Reward anticipation is encoded differently by adolescent ventral tegmental area neurons. Biol Psychiatry. 2016;79:878-86.

19. Allen M, Chowdhury T, Wegener M, Moghaddam B. Efficient sorting of single-unit activity from midbrain cell using KiloSort is as accurate as manualsorting. BioRxiv. 2018;303479. https://doi.org/10.1101/303479.

20. Lohani S, Martig AK, Underhill SM, DeFrancesco A, Roberts MJ, Rinaman L, et al. Burst activation of dopamine neurons produces prolonged post-burst availability of actively released dopamine. Neuropsychopharmacology. 2018;43:2083-92.

21. Matthews M, Bondi C, Torres G, Moghaddam B. Reduced presynaptic dopamine activity in adolescent dorsal striatum. Neuropsychopharmacology. 2013;38:1344-51.

22. Heeringa MJ, Abercrombie ED. Biochemistry of somatodendritic dopamine release in substantia nigra: an in vivo comparison with striatal dopamine release. J Neurochem. 1995;65:192-200.

23. Roth ME, Carroll ME. Sex differences in the acquisition of IV methamphetamine self-administration and subsequent maintenance under a progressive ratio schedule in rats. Psychopharmacology. 2004;172:443-9.

24. Diekhof EK, Keil M, Obst KU, Henseler I, Dechent $P$, Falkai $P$, et al. A functional neuroimaging study assessing gender differences in the neural mechanisms underlying the ability to resist impulsive desires. Brain Res. 2012;1473:63-77.

25. Munro CA, McCaul ME, Wong DF, Oswald LM, Zhou Y, Brasic J, et al. Sex differences in striatal dopamine release in healthy adults. Biol Psychiatry. 2006;59:966-74.

26. Lynch WJ. Sex differences in vulnerability to drug self-administration. Exp Clin Psychopharmacol. 2006;14:34-41.

27. Fattore L, Altea S, Fratta W. Sex differences in drug addiction: a review of animal and human studies. Women's Health (Lond). 2008:4:51-65.

28. Becker JB, Perry AN, Westenbroek C. Sex differences in the neural mechanisms mediating addiction: a new synthesis and hypothesis. Biol Sex Differ. 2012;3:14.

29. Cahill L. Why sex matters for neuroscience. Nat Rev Neurosci. 2006;7:477-84.

30. Konradi C, Kornhuber J, Sofic E, Heckers S, Riederer P, Beckmann H. Variations of monoamines and their metabolites in the human brain putamen. Brain Res. 1992;579:285-90.

31. Laasko A, Vilkman H, Bergman J, Haaparanta $M$, Hietala J. Sex differences in striatal presynaptic dopamine synthesis capacity in healthy subjects. Biol Psychiatry. 2002;52:759-63.

32. Mozley LH, Gur RC, Mozley PD, Gur RE. Striatal dopamine transporters and cognitive functioning in healthy men and women. Am J Psychiatry. 2001;158:1492-9.

33. Gorski RA, Gordon JH, Shryne JE, Southam AM. Evidence for a morphological sex difference within the medial preoptic area of the rat brain. Brain Res. 1978;148:333-46.

34. Caine SB, Bowen CA, Yu G, Zuzga D, Negus SS, Mello NK. Effect of gonadectomy and gonadal hormone replacement on cocaine self-administration in female and male rats. Neuropsychopharmacology. 2004;29:929-42.

35. Forgie ML, Stewart J. Sex differences in the locomotor-activating effects of amphetamine: role of circulationg testosterone in adulthood. Physiol Behevior. 1994;55:639-44.

36. Bhatt SD, Dluzen DE. Dopamine transporter function differences between male and female CD-1 mice. Brain Res. 2005;1035:188-95.

37. McArthur S, McHale E, Gillies GE. The size and distribution of midbrain dopaminergic populations are permanently altered by perinatal glucocorticoid exposure in a sex- region- and time-specific manner. Neuropsychopharmacology. 2007;32:1462-76.

38. Kritzer MF, Creutz LM. Region and sex differences in constituent dopamine neurons and immunoreactivity for intracellular estrogen and androgen receptors in mesocortical projections in rats. J Neurosci. 2008:28:9525-35.

39. Wissman AM, May RM, Woolley CS. Ultrastructural analysis of sex differences in nucleus accumbens synaptic connectivity. Brain Struct Funct. 2012;217:181-90.

40. Cavigelli SA. Animal personality and health. Behaviour. 2005;142:1223-44.
41. Turner KM, Burne TH. Comprehensive behavioural analysis of Long Evans and Sprague-Dawley rats reveals differential effects of housing conditions on tests relevant to neuropsychiatric disorders. PLoS ONE. 2014;9:e93411.

42. Park J, Moghaddam B. Impact of anxiety on prefrontal cortex encoding of cognitive flexibility. Neuroscience. 2017;345:193-202.

43. Lohani S, Martig AK, Deisseroth K, Witten IB, Moghaddam B. Dopamine modulation of prefrontal cortex activity is manifold and operates at multiple temporal and spatial scales. Cell Rep. 2019;27:99-114 e116.

44. Yu Q, Liu YZ, Zhu YB, Wang YY, Li Q, Yin DM. Genetic labeling reveals temporal and spatial expression pattern of D2 dopamine receptor in rat forebrain. Brain Struct Funct. 2019:224:1035-49.

45. Chowdhury TG, Wallin-Miller KG, Rear AA, Park J, Diaz V, Simon NW, et al. Sex differences in reward- and punishment-guided actions. Cogn Affect Behav Neurosci. 2019;19:1404-17.

46. Becker JB, Prendergast BJ, Liang JW. Female rats are not more variable than male rats: a meta-analysis of neuroscience studies. Biol Sex Differ. 2016;7:34.

47. Prendergast BJ, Onishi KG, Zucker I. Female mice liberated for inclusion in neuroscience and biomedical research. Neurosci Biobehav Rev. 2014;40:1-5.

48. Scholl JL, Renner KJ, Forster GL, Tejani-Butt S. Central monoamine levels differ between rat strains used in studies of depressive behavior. Brain Res. 2010;1355:41-51.

49. Swerdlow NR, Martinez ZA, Hanlon FM, Platten A, Farid M, Auerbach $P$, et al. Toward understanding the biology of a complex phenotype: rat strain and substrain differences in the sensorimotor gating-disruptive effects of dopamine agonists. J Neurosci. 2000;20:4325-36.

50. Zamudio S, Fregoso T, Miranda A, De La Cruz F, Flores G. Strain differences of dopamine receptor levels and dopamine related behaviors in rats. Brain Res Bull. 2005;65:339-47.

51. Glowinski J. Properties and functions of intraneuronal monoamine compartments in central aminergic neurons. In: Iversen, L. L., Iversen, S. D., Snyder, S. H. (eds) Biochemistry of Biogenic Amines. Handbook of Psychopharmacology (Section I: Basic Neuropharmacology), vol 3. Springer, Boston, MA, 1975.

52. Naudon L, Leroux-Nicollet I, Raisman-Vozari R, Botton D, Costentin J. Time-course of modifications elicited by reserpine on the density and mRNA synthesis of the vesicular monoamine transporter, and on the density of the membrane dopamine uptake complex. Synapse. 1995;21:29-36.

53. Martin-Iverson MT, Yamada N, By AW, Lodge BA. "Designer" amphetamines: effects on behavior and monoamines with or without reserpine and/or alphamethyl-para-tyrosine pretreatment. J Psychiatry Neurosci. 1991;16:253-61.

54. Bernstein Al, Stout KA, Miller GW. The vesicular monoamine transporter 2: an underexplored pharmacological target. Neurochem Int. 2014;73:89-97.

55. Yuan J, Cord BJ, McCann UD, Callahan BT, Ricaurte GA. Effect of depleting vesicular and cytoplasmic dopamine on methylenedioxymethamphetamine neurotoxicity. J Neurochem. 2002;80:960-9.

56. Sabol KE, Seiden LS. Reserpine attenuates D-amphetamine and MDMA-induced transmitter release in vivo: a consideration of dose, core temperature and dopamine synthesis. Brain Res. 1998;806:69-78.

57. Biggs CS, Fowler LJ, Whitton PS, Starr MS. NMDA receptor antagonists increase the release of dopamine in the substantia nigra of reserpine-treated rats. Eur J Pharm. 1996;299:83-91.

58. Moghaddam B, Roth RH, Bunney BS. Characterization of dopamine release in the rat medial prefrontal cortex as assessed by in vivo microdialysis: comparison to the striatum. Neuroscience. 1990;36:669-76.

59. Cadoni C, Pinna A, Russi G, Consolo S, Di Chiara G. Role of vesicular dopamine in the in vivo stimulation of striatal dopamine transmission by amphetamine: evidence from microdialysis and Fos immunohistochemistry. Neuroscience. 1995;65:1027-39.

60. Abercrombie ED, Keefe KA, DiFrischia DS, Zigmond MJ. Differential effect of stress on in vivo dopamine release in striatum, nucleus accumbens, and medial frontal cortex. J Neurochem. 1989;52:1655-8.

61. Sulzer D, Chen TK, Lau YY, Kristensen H, Rayport S, Ewing A. Amphetamine redistributes dopamine from synaptic vesicles to the cytosol and promotes reverse transport. J Neurosci. 1995;15:4102-8.

62. Virdee K, McArthur S, Brischoux F, Caprioli D, Ungless MA, Robbins TW, et al. Antenatal glucocorticoid treatment induces adaptations in adult midbrain dopamine neurons, which underpin sexually dimorphic behavioral resilience. Neuropsychopharmacology. 2014;39:339-50.

63. Calipari ES, Ferris MJ. Amphetamine mechanisms and actions at the dopamine terminal revisited. J Neurosci. 2013;33:8923-5. 